\title{
Demonstration of superprism effect in silicon pillar 2-D photonic crystal infiltrated with liquid crystals
}

\author{
Pierre-Yves Baroni \\ Vincent Paeder \\ Yu-Chi Chang \\ Matthieu Roussey \\ Hans Peter Herzig \\ Ecole Polytechnique Fédérale de Lausanne \\ Institute of Microengineering \\ Optics and Photonics Laboratory \\ A.-L. Breguet 2 \\ 2000 Neuchâtel, Switzerland \\ E-mail: matthieu.roussey@epfl.ch
}

\section{Wataru Nakagawa}

Montana State University, Bozeman

Electrical and Computer Engineering Department

Montana 59717-3780

\begin{abstract}
Superprism-based deflection of an optical beam is observed in a photonic crystal composed of a triangular lattice of pillars infiltrated with a liquid crystal. The device is based on a Silicon-on-insulator substrate and operates in the telecommunications band. The experimental results show a wavelength shift of $0.76 \mu \mathrm{m} / \mathrm{nm}$, in reasonable agreement with simulations. Temperature-based control of the liquid crystal properties is also shown to modulate the superprism characteristics. (C) 2011 Society of Photo-Optical Instrumentation Engineers (SPIE). [DOI: 10.1117/1.3529437]
\end{abstract}

Subject terms: nanophotonics; liquid crystals; photonics; submicron lithography; thermal effects.

Paper 100570R received Jul. 14, 2010; revised manuscript received Nov. 10, 2010; accepted for publication Nov. 17, 2010; published online Jan. 18, 2011.

\section{Introduction}

One of the interesting optical properties of photonic crystals $(\mathrm{PhCs})^{1,2}$ is the so-called superprism effect, ${ }^{3-5}$ producing a strong angular dispersion for frequencies near the edges of the photonic bandgaps. This effect could enable compact, on-chip devices such as multiplexer-demultiplexers ${ }^{6-8}$ or ultrafast switches. ${ }^{9}$ In particular, a tunable superprism device would be very useful for such applications, and a number of tuning mechanisms have been explored, including electrooptic $^{10,11}$ or mechanical ${ }^{12}$ control, nonlinear materials, ${ }^{13,14}$ or infiltration with liquid crystals. ${ }^{15-19}$ In this paper, we investigate planar triangular-lattice $\mathrm{PhC}$ structures made of pillars and infiltrated with liquid crystals to realize a tunable superprism effect.

The devices are fabricated using a standard e-beam lithography process on a silicon-on-insulator (SOI) wafer. The structure under study is shown in Fig. 1. It is composed of an input waveguide followed by a $\mathrm{PhC}$ region whose orientation is rotated by $15 \mathrm{deg}$ clockwise relative to the input waveguide. A planar output waveguide conducts light to the edge of the device. The $\mathrm{PhC}$ is composed of a triangular lattice of pillars with a period of $860 \mathrm{~nm}$, height $340 \mathrm{~nm}$, and diameter $602 \mathrm{~nm}$. The structure is filled with liquid crystals (5CB from Merck) having refractive indices at $\lambda=1500 \mathrm{~nm}$ of $n_{o}=1.51$ and $n_{e}=1.63$ at room temperature and $n_{i}=1.57$ (isotropic) above $36^{\circ} \mathrm{C}$.

\section{Modeling and Theory}

A theoretical analysis of the $\mathrm{PhC}$ structure has been conducted using the MIT Photonic Bands (MPB) ${ }^{20}$ software. Isofrequency contours (IFC) for band 5 (TM polarization) are shown in Fig. 2. They highlight two different superimposed effects. At longer wavelengths, a superprism effect is obtained due to the large curvature of the IFC. Moving toward shorter wavelengths, a forbidden band appears, caused by the transition of the incident medium IFC through the Brillouin

0091-3286/2011/\$25.00 @ 2011 SPIE zone (BZ) boundary. ${ }^{21}$ This effect is entirely determined by the period of the structure. Since the incident medium IFC is large compared to the $\mathrm{BZ}$, it is folded multiple times in the BZ. The incident wavevector can lie on any of the foldings depending on the angle of incidence and the frequency. By carefully choosing the angle, the wavevector can be set close to the edge of the BZ. Varying the frequency hence makes the wavevector hop from one folding to another, mimicking the presence of a bandgap. Below this gap, the incident wave matches a second region with super-refracting properties.

From the IFC, we extract the refraction angle at the $\mathrm{PhC}$ interface. This angle is presented in Fig. 3 for three values of the liquid crystal refractive index. The two discrete regions where the slope of the curves is greatest indicate a superprism effect for two of the refractive index values. In contrast, for the extraordinary index $\left(\mathrm{n}_{e}=1.63\right)$, only one region exhibits a superprism effect.

Two-dimensional finite integral time domain (FITD) simulations with effective index approximation have been performed using CST Microwave Studio. As expected from the MPB simulations, a superprism effect is present as shown in Fig. 4. A displacement of the output spot of $30 \mu \mathrm{m}$ is obtained (solid line) corresponding to a displacement rate of $1.2 \mu \mathrm{m} / \mathrm{nm}$. Note that the origin of the output spot displacement scale is defined to be the center of the input waveguide. The dashed curve shows the output spot intensity. Large insertion losses, as described in other work, ${ }^{22,23}$ are particularly visible. At longer wavelengths, the intensity of the light coupled to the edge of the fourth $\mathrm{PhC}$ band is seen to overwhelm the effect of interest. Note that according to MPB, the fourth band lies outside the investigated frequency range. However, beam dispersion at the output of the waveguide and finite size effects can account for the small fraction of light transmitted in the fourth band.

\section{Experimental Results}

In the experiment, light from a tunable laser (1460-1590 nm) is polarized in a TM mode and injected into the waveguide through a lensed fiber (see Fig. 5). The detection is performed 


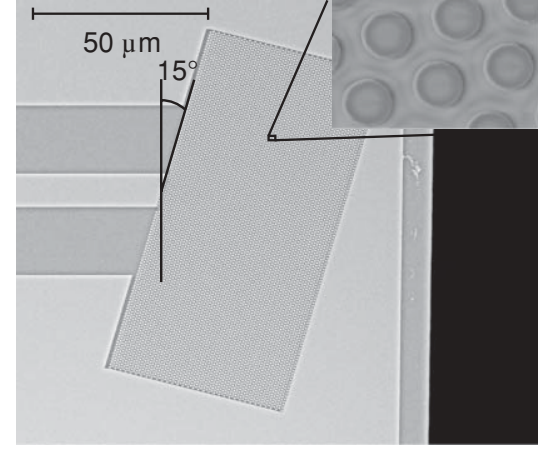

Fig. 1 SEM picture of the superprism device fabricated on a SOI wafer. Incident light arrives from the left in the ridge waveguide, then encounters the PhC structure rotated by 15 with respect to the waveguide axis.
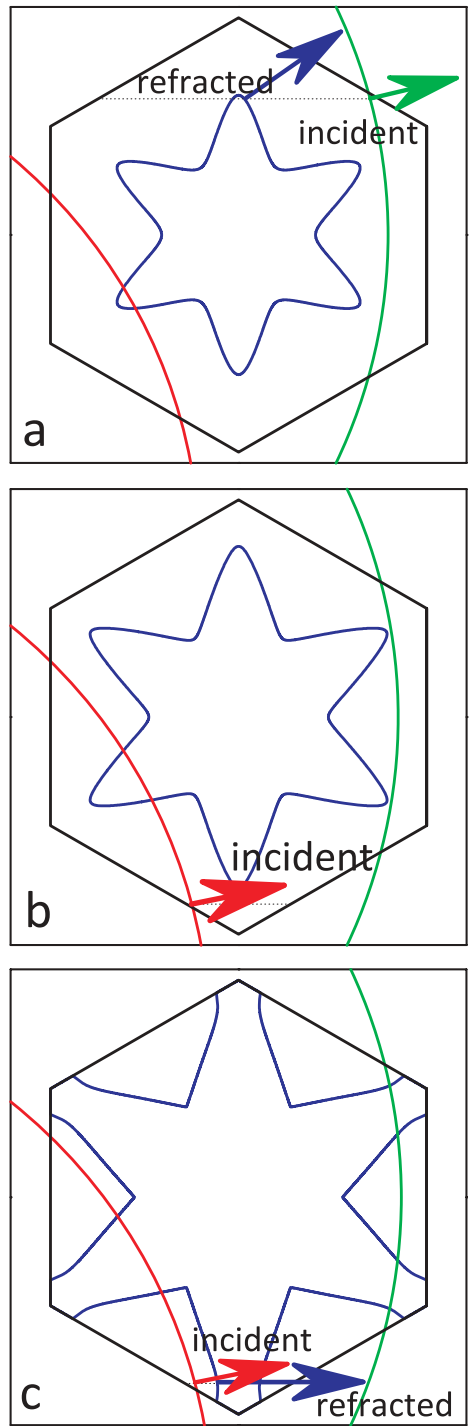

Fig. 2 Isofrequency contours at three different wavelengths; a) $\lambda=1522 \mathrm{~nm}$ (superprism regime), b) $\lambda=1494 \mathrm{~nm}$ (forbidden band), c) $\lambda=1463 \mathrm{~nm}$ (superprism regime). The red and green curves represent two translated copies of the IFC in the incident medium.

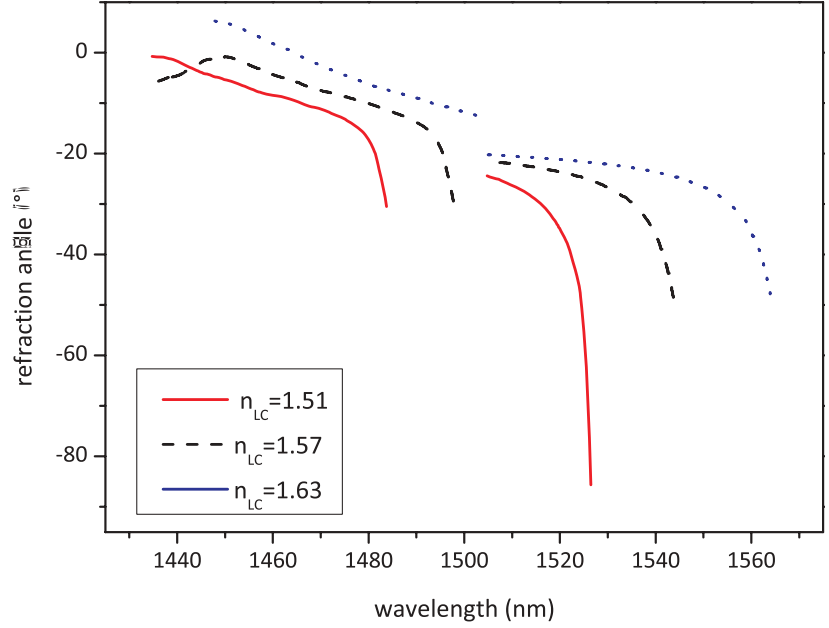

Fig. 3 Refraction angle at the waveguide-PhC interface for three refractive indices of the liquid crystals, computed using MPB. With $n_{o}=1.51, n_{i}=1.57$, and $n_{e}=1.63$ the ordinary, isotropic, and extraordinary refractive indices of the liquid crystal at $\lambda=1500 \mathrm{~nm}$.

by imaging the output edge using an InGaAs infrared camera (Polytec model SU320MS-1.7RT) positioned in front of the planar output waveguide. Another IR camera is mounted above the sample in order to precisely align the injection into the planar waveguide. The device is designed to work with liquid crystal surrounding the structure, so, a droplet of liquid crystal is deposited on the $\mathrm{PhC}$.

The experimental result (see Fig. 6) shows two distinct regions where a superprism effect is observed (emphasized by the red lines). Note that in Fig. 7 the origin of the output spot displacement is arbitrary. The region included between $\lambda=1493 \mathrm{~nm}$ and $\lambda=1515 \mathrm{~nm}$ exhibits a displacement rate of $0.55 \mu \mathrm{m} / \mathrm{nm}$. The greatest displacement, equal to $20.5 \mu \mathrm{m}$, is measured between $\lambda=1541 \mathrm{~nm}$ and $\lambda=1568 \mathrm{~nm}$ and corresponds to a displacement rate of $0.76 \mu \mathrm{m} / \mathrm{nm}$, which corresponds to a deviation of $0.6^{\circ} / \mathrm{nm}$. As a comparison, a conventional prism has a deviation around $0.006^{\circ} / \mathrm{nm}$ and a diffraction grating $0.1^{\circ} / \mathrm{nm}^{24,25}$

The measured values are smaller than the ones predicted by the FITD model. This difference could arise from the dif-

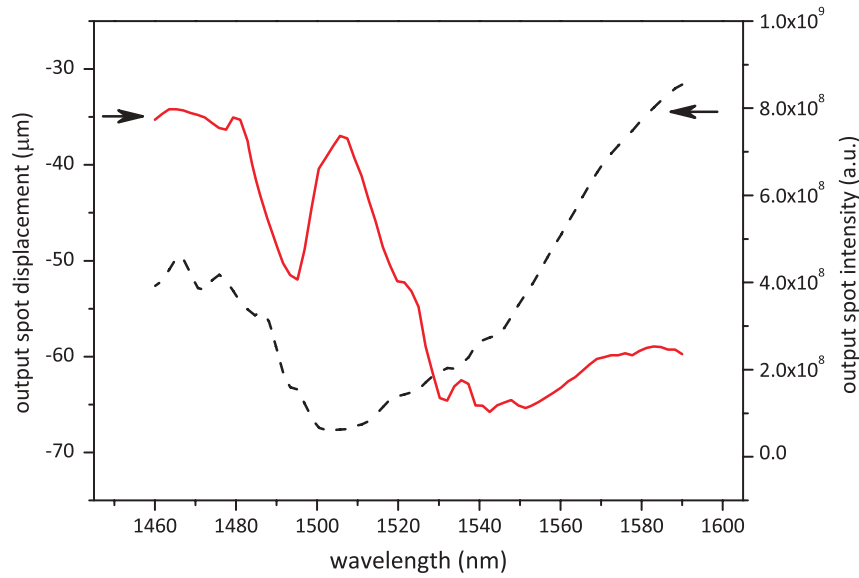

Fig. 4 Two-dimensional simulation of the structure showing an anomalous dispersion (red curve) and the related intensity of the output spot (black dashed curve). 


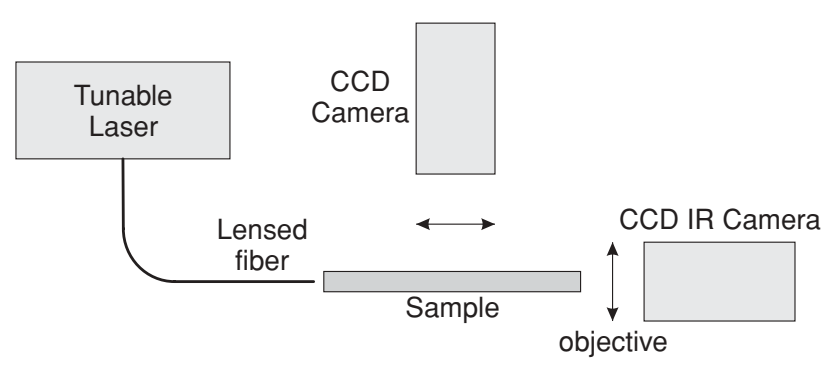

Fig. 5 Scheme of the experimental setup.

ficulty of detecting the adjacent sharp dip and peak observed in Fig. 7. We also observe a red shift of the area of interest between the simulations and the experiment that could be due to imperfect alignment of the liquid crystal with respect to the pillars. Nevertheless, the superprism effect is presented for the first time for a $\mathrm{PhC}$ made of silicon pillars infiltrated by liquid crystal.

The superprism properties can be modulated using temperature, due to the fact that the optical properties of the liquid crystal change from anisotropic to isotropic at $36^{\circ} \mathrm{C}$. In this experiment, a Pelletier element is used to heat the device and liquid crystal from room temperature to $45^{\circ} \mathrm{C}$. Figure 7 shows the images, obtained by the in-plane camera, of the spot refracted by the PhC at a wavelength of $\lambda=1510 \mathrm{~nm}$. Figures 7(a) and 7(c) show the spots before and after heating, whereas Fig. 7(b) shows the same area with the liquid crystal in the anisotropic state. [The intensity variation between the spots shown in Figs. 7(a) and 7(c) is most likely explained by the automatic calibration of the infrared camera.] The disappearance of the spot at the higher temperature is due to the extreme sensitivity of the superprism effect to the surrounding refractive index. This slow temperaturebased modulation could be enhanced by instead using the electro-optic properties of the liquid crystal. Moreover, the liquid crystal infiltration adds functionality that makes this structure promising for future generations of very compact optical systems.

\section{Conclusion}

We have simulated, fabricated, and characterized a device exhibiting a superprism effect in a two dimensional $\mathrm{PhC}$

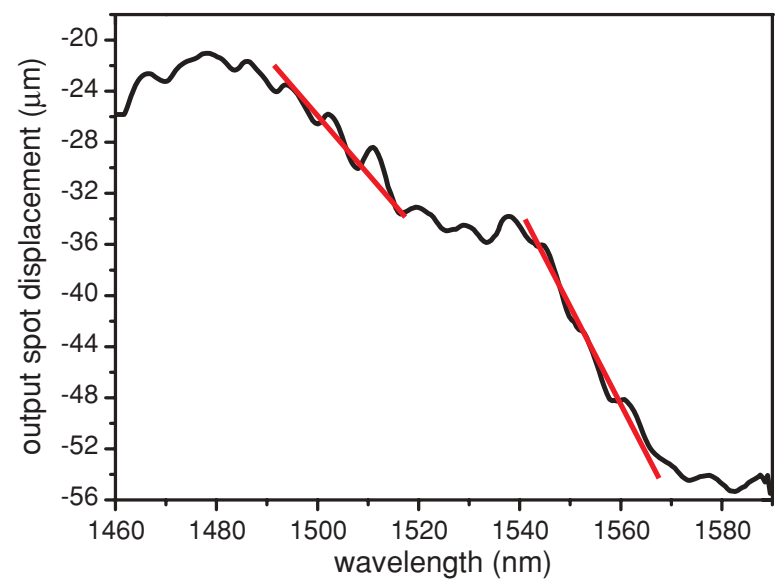

Fig. 6 Experimental result demonstrating a superprism effect in the infrared range using silicon pillars surrounded by liquid crystal. The red lines show the regions where the effect is obtained.
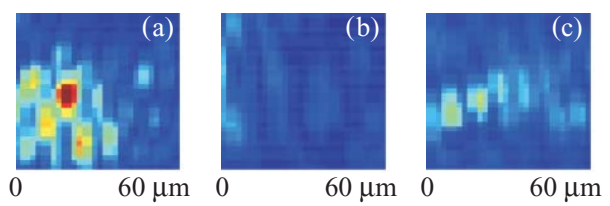

Fig. 7 Temperature-based modulation of the superprism device: output light spot at room temperature (a) initially, (b) after heating to $45^{\circ} \mathrm{C}$, and (c) after returning to room temperature. Note that in (b) no output spot is observed due to the change in the liquid crystal index of refraction.

composed of silicon pillars infiltrated by liquid crystal. We achieve a $0.76 \mu \mathrm{m}$ shift per nm of injected wavelength in the near infrared. An improved time response and control of the orientation of the liquid crystal could be achieved through electro-optic modulation. Further improvements could be based on manufacturing by interference lithography, which would allow efficient production at very low cost.

\section{References}

1. E. Yablonovitch, Phys. Rev. Lett. 58, 2059 (1987).

2. S. John, Phys. Rev. Lett. 58, 2486 (1987).

3. H. Kosaka et al., Phys. Rev. B 58, 10096 (1998)

4. S.-Y. Lin, V. M. Hietala, L. Wang, and E. D. Jones, Opt. Lett. 21, 1771 (1996).

5. T. Baba, T. Matsumoto, and M. Echizen, Opt. Express 12, 4608 (2004).

6. A. Jugessur et al., Opt. Express 14, 1632 (2006).

7. K. B. Chung and S. W. Hong, App. Phys. Lett. 81, 1549 (2002).

8. D. Bernier et al., Opt. Express 16, 17209 (2008).

9. J. Amet, F. I. Baida, G. W. Burr, and M.-P. Bernal, Photonics Nanostruct. Fundam. Appl. 6, 47 (2008).

10. D. Scrymgeour, N. Malkova, S. Kim, and V. Gopalan, Appl. Phys. Lett. 82, 3176 (2003).

11. S. Xiong and H. Fukshima, J. Appl. Phys. 94, 1286 (2003).

12. I. Märki, M. Salt, and H. P. Herzig, Opt. Express 14, 2969-2978 (2006).

13. N. C. Panoiu, M. Bahl, and R. M. Osgood, Jr., Opt. Lett. 28, 2503 (2003).

14. S. Diziain, J. Amet, F. I. Baida, and M.-P. Bernal, Appl. Phys. Lett. 93, 261103 (2008).

15. J. Martz, R. Ferrini, F. Nüesch, L. Zuppiroli, B. Wild, L. A. Dunbar, R. Houdr, M. Mulot, and S. Anand, J. Appl. Phys. 99, 103105 (2006).

16. R. Ferrini, J.Martz, L. Zuppiroli, B. Wild, V. Zabelin, L. A. Dunbar, R. Houdr, M. Mulot, and S. Anand, Opt. Lett. 31, 1238-1240 (2006).

17. P. El-Kallassi, R. Ferrini, L. Zuppiroli, N. Le Thomas, R. Houdr, A. Berrier, S. Anand, and A. Talneau, J. Opt. Soc. Am. B 24, 21652171 (2007).

18. G. Alagappan, X. W. Sun, P. Shum, and M. B. Yu, Opt. Lett. 31, 1109 (2006).

19. G. Alagappan, X.W. Sun, M. B. Yu, P. Shum, and D. Den Engelsen, IEEE J. Quantum Electron. 42, 404 (2006).

20. S. G. Johnson and J. D. Joannopoulos, Opt. Express 8, 173 (2001).

21. A. Lupu et al., Opt. Express 14, 2003 (2006).

22. J. Witzens, M. Hochberg, T. Baehr-Jones, and A. Scherer, Phys. Rev. E 69, 046609 (2004).

23. F. J. Lawrence, L.C. Botten, K. B. Dossou, and C. M. de Sterke, Appl. Phys. Lett. 93, 121114 (2008).

24. A. Thorne, U. Litzn, and S. Johansson, Spectrophysics: Principles and Applications, Springer-Verlag Berlin, 1999.

25. F. Glöckler, S.Peters, U. Lemmer, and M. Gerken, Phys. Status Solidi A 204, 3790-3804 (2007).

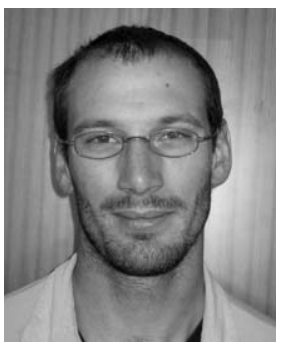

Pierre-Yves Baroni studied microtechnics at the Ecole Polytechnique Fédérale de Lausanne (EPFL) from where he received his diploma in 2003. He was a member of the optics group of the University of Neuchâtel and of the EPFL between 2004 and 2009. He worked in the field of periodic silicon nanostructures and their interactions with the light. He received his PhD in 2010. 


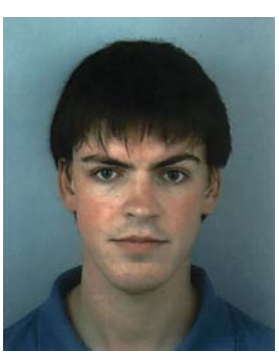

Vincent Paeder received the MS degree from the University of Neuchâtel, Switzerland, in 2006, and is now working toward the PhD in the Optics and Photonics Technology Laboratory of the Ecole Polytechnique Fédérale de Lausanne (EPFL). His current research interests include the simulation of nanostructured optical devices.

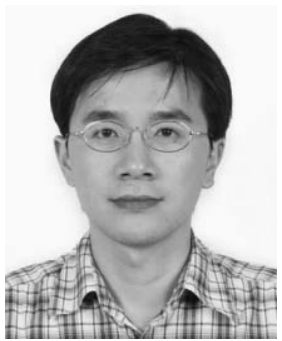

Yu-Chi Chang received the BSc in Physics from National Taiwan University, Taipei, Taiwan, in 1998, and the MSc in Optical Sciences from National Central University, Jhongli, Taiwan, in 2002. From 2002 to 2006 , he was an engineer with the TPO Display Corporation, Taiwan, working in Liquid Crystal Display design and technology development. Currently, he is a PhD student of the Optics and Photonics Technology laboratory, Ecole Polytechnique Fdrale de Lausanne (EPFL). His research areas are mid-infrared waveguide sensors and silicon photonics.

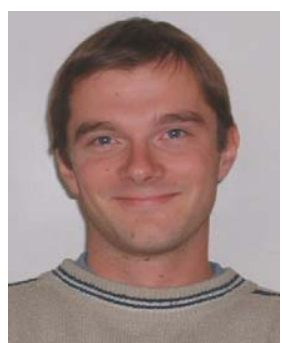

Matthieu Roussey received the $\mathrm{PhD}$ degree in optics from the University of FrancheComt in 2007 at the institute FEMTO-ST (Besançon, France), on the subject of tunable photonic crystals in lithium niobate. $\mathrm{He}$ joined the Institut of Microtechnique in 2007 and is currently project leader in the Optics and Photonics Technology Laboratory of the Ecole Polytechnique Fdrale de Lausanne, in Neuchtel. His research interests are in the nano-optics engineering field including the simulation, fabrication, and characterization of optical nano-structures.

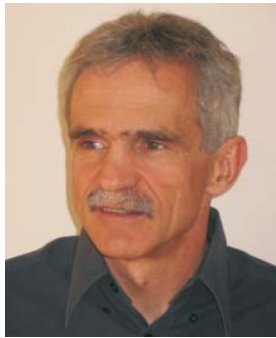

Hans Peter Herzig received the Diploma degree in physics from the Swiss Federal Institute of Technology, Zurich, Switzerland, in 1978 , and the PhD degree in optics from the University of Neuchtel, in 1987. From 1978 to 1982 , he was a scientist with the Department of Optics Development of Kern, Aarau, Switzerland, working in lens design and optical testing. In 1983, he was a graduate research assistant with the Applied Optics Group, Institute of Microtechnology, University of Neuchtel, Switzerland, working in the field of holographic optical elements. From 1989 to 2001, he was the head of the MicroOptics Research Group. From 2002 to 2008, he was a full professor and the head of the Applied Optics Laboratory, University of Neuchtel. He joined the faculty with the cole Polytechnique Fdrale de Lausanne (EPFL), Switzerland, in 2009. Currently, he is a professor with the EPFL and (2009/2010) President of the European Optical Society (EOS). His current research interests include refractive and diffractive micro-optics, nano-scale optics, and optical MEMS. Dr. Herzig is a member of the OSA, IEEE Photonics Society, EOS, and SSOM. He served as the Conference Chairman for international conferences of EOS, IEE, IEEE/LEOS, OSA, and SPIE, and as a guest editor of four special issues of IEEE and OSA journals. He is the editor of a well-known book on micro-optics (published in English and Chinese) and author of 12 book chapters, over 110 journal papers, and 240 conference proceedings.

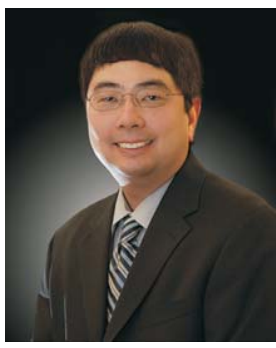

Wataru Nakagawa received his BS degree in physics from Stanford University in 1996 and his MS and PhD degrees in Electrical Engineering (Applied Physics) from the University of California, San Diego, in 1999 and 2002 , respectively. He is currently an assistant professor of Electrical and Computer Engineering at Montana State University, in Bozeman, MT. His research interests include near-field optical effects in nanostructures, the development of photonic devices based on these phenomena, and the interdisciplinary applications of such devices. He is a member of OSA and a senior member of the IEEE Photonics Society. 